\title{
AVALIAÇÃO DOS EFEITOS DE INTERFERÊNCIAS NO DESEMPENHO DE SISTEMAS DE COMUNICAÇÕES DIGITAIS *
}

\author{
José Mauro P. Fortes e Raimundo Sampaio Neto \\ Pontifícia Universidade Católica do Rio de Janeiro \\ Rua Marquês de São Vicente, 225 \\ Gávea, Rio de Janeiro - RJ 22453 \\ E-mail : jmfortes@mamao.cetuc.puc-rio.br
}

\begin{abstract}
Resumo : Este trabalho examina, compara e propõe técnicas analíticas para a avaliação dos efeitos de interferências em sistemas de comunicações digitais. Com relação aos efeitos na probabilidade de erro de símbolo, o desenvolvimento concentra-se na técnica que utiliza o limitante de Glave e Rosembaum. Em sua versão original, a aplicação deste limitante restringe-se ao caso de portadoras PSK Este trabalho estende a aplicabilidade do limitante a sistemas com modulação QAM. Além disso, os resultados apresentados utilizam um algoritmo original desenvolvido para a determinação analítica do valor de pico de portadoras QAM-16 filtradas, parâmetro este necessário para o cálculo do limitante quando os sinais interferentes incluem este tipo de portadora. Um novo conceito de ruí do térmico equivalente é proposto para a obtenção de estimativas da taxa de erro de bit do sistema a partir do conhecimento da probabilidade de erro de símbolo em presença de interferências e da curva de desempenho do modem em presença de ruído. A aplicação das técnicas abordadas é ilustrada através de exemplos numéricos.
\end{abstract}

Abstract: This work examines analytical techniques to assess the effects of interferences on the performance of digital transmission systems. To evaluate the effect of interference in the symbol error probability the upper bound proposed by Glave \& Rosembaum is used. The use of this upper bound, originally proposed for PSK modulation systems is extended to include QAM systems. The key parameter for the evaluation of the Glave \& Rosembaum upper bound is the interfering signal peak value after filtering by the receiver detection filter. An original analytical algorithm to determine this parameter in the case of QAM-16 interfering signals is presented. In order to obtain estimates of the system bit error rate performance in the presence of interfering signals, a new concept of equivalent noise is proposed. This concept is used to generate estimates of the $B E R$ from the knowledge of the system theoretical symbol error probability in the presence of interferences and the system modem performance curves in the presence of background noise only. The application of the described techniques is illustrated by examples.

Palavras chaves : interferência, probabilidade de erro, limitante de Glave e Rosembaum.

\footnotetext{
* Este trabalho foi desenvolvido com suporte da Embratel sob os Aditivos de Contrato 01 e 18 do Convênio Embratel/PUC P005/94
}

\section{INTRODUÇÃO}

Diversas situações em telecomunicações requerem que a avaliação dos efeitos de interferências em sistemas se transmissão digitais, além de precisa, seja feita de maneira rápida e eficiente. Este é o caso, por exemplo, do dimensionamento e otimização de transponders em comunicações por satélite. Este trabalho tem como objetivo a investigação de métodos analíticos que forneçam, de forma rápida e eficiente, resultados de desempenho de sistemas de comunicações digitais sujeitos a interferências externas.

Com relação à probabilidade de erro de sí mbolo, um exame de alguns dos métodos propostos para a avaliação dos efeitos de interferências indicou que dois deles possuem maior potencial para satisfazer os objetivos de rapidez e eficiência na obtenção dos resultados : (i) o método que utiliza os momentos da amostra do agregado de sinais interferentes e a regra de quadratura de Gauss na determinação da probabilidade de erro de símbolo e; (ii) o método que utiliza o limitante superior proposto por Glave e Rosembaum para a probabilidade de erro de símbolo, obtido analiticamente. Ambos os métodos são abordados na Seção 4. deste trabalho, que, entretanto, concentra-se naquele que utiliza o limitante de Glave e Rosembaum considerado como mais adequado para os objetivos propostos. No entanto este limitante, conforme proposto em [1, 2], contempla apenas o desempenho de sistemas PSK. O presente trabalho estende a aplicabilidade deste limitante a sistemas com modulação QAM, tendo em vista a tendência atual de utilização deste tipo de modulacão em sistemas de comunicações por satélite. Ressalte-se ainda que a determinação do limitante de Glave e Rosembaum requer, em todos os casos, a determinação do valor de pico do agregado de sinais interferentes, após filtragem pelo filtro de recepção. Em $[1,2]$, é proposto um algoritmo para a determinação analítica do valor de pico de sinais PSK M-ários filtrados, que não se aplica, entretanto, ao caso mais complexo de sinais interferentes QAM M-ários.Um algoritmo original para a determinação do valor de pico de sinais QAM-16 filtrados foi então desenvolvido e aplicado na avaliação dos efeitos de portadoras interferentes QAM-16 em sistemas com modulação PSK ou QAM. Estes algoritmos são apresentados no Apêndice.

Na seção 5. são apresentados alguns resultados numéricos para a probabilidade de erro de símbolo envolvendo portadoras PSK e QAM obtidos com a utilização do limitante de Glave e Rosembaum e comparações com aqueles obtidos com 
o método que utiliza a regra de quadratura de Gauss.

Os efeitos de interferências na taxa de erro de bit do sistema de transmissão digital, que pode ou não utilizar códigos corretores de erro, é considerado na Seção 6. onde um novo conceito de ruído térmico equivalente é proposto para a obtenção de estimativas da taxa de erro de bit do sistema a partir do conhecimento da probabilidade de erro de símbolo em presença de interferências e da curva de desempenho do modem em presença de ruído. A aplicação destas técnicas é ilustrada na Seção 7. através de exemplos envolvendo situações práticas.

\section{CARACTERIZAÇÃO DO RECEP- TOR E DOS SINAIS RECEBIDOS}

A caracterização apresentada baseia-se no modelo matemático equivalente associado ao receptor de sinais digitais com modulação em amplitude e fase, apresentado na Figura 1 , que lida com as envoltórias complexas dos sinais. Nesta figura $\tilde{r}(t)$ caracteriza a envoltória complexa do sinal recebido na saída do filtro de deteção $h(t)$, dado por

$$
\tilde{r}(t)=\tilde{s}(t)+\tilde{u}(t)+\tilde{n}(t)
$$

onde, supondo-se recuperação ideal de portadora,

$$
\tilde{s}(t)=\sqrt{2 C} \sum_{k=-\infty}^{\infty} a_{k} p(t-k T)
$$

é a envoltória complexa do sinal desejado, com $C$ denotando a potência média do sinal em RF, $T$ o inverso da taxa de símbolos $R_{s}$ e $\left\{a_{k}\right\}$ é uma seqüencia de complexos que assumem valores na constelação de sinais da modulação. O pulso $p(t)$ é dado por

$$
p(t)=v(t) * h(t)
$$

onde $v(t)$ é o pulso de transmissão, em geral complexo, normalizado de tal forma que

$$
\frac{1}{T} \int_{-\infty}^{\infty}|v(t)|^{2} d t=1
$$

e o símbolo “*”denota convolução.

Em (1), $\tilde{n}(t)$ é a envoltória complexa de um ruí do aditivo gaussiano branco com nível espectral $N_{0} / 2$ filtrado pelo filtro de recepção e $\tilde{u}(t)$ é a envoltória complexa da interferência passa-faixa após filtragem, que pode ser expressa na forma geral

$$
\tilde{u}(t)=u(t): e^{j\left(\phi(t)+\theta_{n}\right)}
$$

$\operatorname{com} \theta_{u}$ representando uma variável aleatória uniformemente distribuída em $(0,2 \pi]$. Considerando-se, sem perda de generalidade, a deteção do sí mbolo de índice $k=0\left(a_{0}\right)$ e supondo a ausência de interferência entre símbolos na amostra do sinal desejado, tem-se que

$$
\tilde{r}_{0}=\tilde{r}\left(t_{0}\right)=\sqrt{2 C} a_{0} p_{0}+u e^{j \xi}+\tilde{n}
$$

onde $p_{0}=\left|p\left(t_{0}\right)\right|, \tilde{n}=\tilde{n}\left(t_{0}\right), u=u\left(t_{0}\right)$ e $\xi=\phi\left(t_{0}\right)+\theta_{u}$. Note que como $\theta_{u}$ é uma variável aleatória uniformemente 86

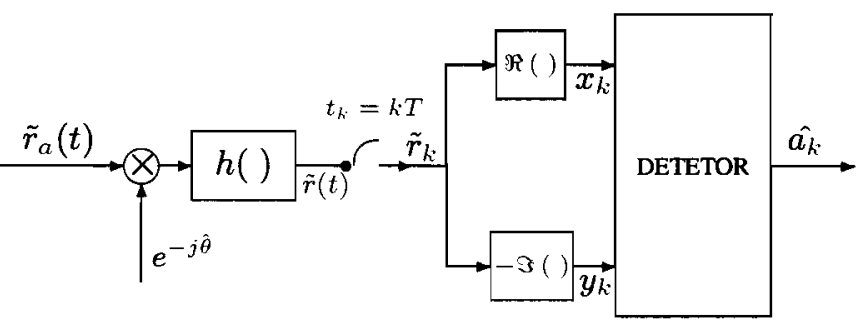

FIG. 1: Modelo Matemático Equivalente Associado ao Receptor de Sinais Digitais com Modulação em Amplitude e Fase.

distribuída em $(0,2 \pi]$ e independente de $\phi\left(t_{0}\right)$ e $u\left(t_{0}\right)$, a variável aleatória $\xi$, definida módulo $2 \pi$, será também uniformemente distribuída e estatisticamente independente de $u$. Esta propriedade caracteriza a interferência $\tilde{u}$ como sendo Circularmente Simétrica.

\section{PROBABILIDADE DE ERRO DE SÍMBOLO EM PRESENÇA DE INTERFERÊNCIA CIRCULAR- MENTE SIMÉTRICA}

Esta seção considera sistemas com modulaç ão PSK e QAM com transmissão em canal AWGN (Additive White Gaussian Noise), em presença de interferência externa Circularmente Simétrica. São apresentadas expressões para a probabilidade de erro de símbolo condicionada a um dado valor da amostra de interferência.

No caso de portadoras BPSK, com $a_{0}= \pm 1$, é possível mostrar que a probabilidade de erro de símbolo condicionada a um dado valor da amostra de interferência $(u, \xi)$, pode ser expressa na forma [3]

$$
P_{s}(e)=E_{u, \xi}\left[Q\left(\sqrt{2 \gamma}+\sqrt{\frac{2 \gamma}{\beta}} \frac{u}{\mu_{u}} \cos \xi\right)\right]
$$

onde $E[::]$ denota o operador valor esperado, $Q(::)$ é a função definida por

$$
Q(x)=\frac{1}{\sqrt{2 \pi}} \int_{x}^{\infty} e^{-\alpha^{2} / 2}: d \alpha
$$

$\gamma$ é a razão portadora-ruído (cnr) na saída do filtro de deteção, definida por

$$
\gamma=\frac{E\left[\left|\tilde{s}\left(t_{0}\right)\right|^{2}\right]}{E\left[\left|\tilde{n}\left(t_{0}\right)\right|^{2}\right]}
$$

e $\beta$ é a razão portadora-interferência (cir) na saída do filtro de deteção, definida por

$$
\beta=\frac{E\left[\left|\tilde{s}\left(t_{0}\right)\right|^{2}\right]}{E\left[u^{2}\right]}
$$

Em (7), $\mu_{u}=\sqrt{E\left[u^{2}\right]}$ representa o valor rms da amplitude do sinal interferente na saída do filtro de deteção. 
No caso mais geral de portadoras MPSK, considerando-se a condição de interferência circularmente simétrica, é possível obter-se um um limitante superior para a probabilidade de erro de símbolo similar a (7), resultando [3]

$$
P_{s}(e) \leq 2 E_{u, \xi}\left[Q\left(\sqrt{2 \gamma_{1}}+\sqrt{\frac{2 \gamma_{1}}{\beta_{1}}} \frac{u}{\mu_{u}} \cos \xi\right)\right]
$$

onde

$$
\begin{aligned}
& \gamma_{1}=\gamma \operatorname{sen}^{2} \frac{\pi}{M} \\
& \beta_{1}=\beta \operatorname{sen}^{2} \frac{\pi}{M}
\end{aligned}
$$

com $\gamma$ e $\beta$ definidos por (9) e (10), respectivamente.

Considerando-se o caso de portadoras MQAM, novamente a condição de interferência circularmente simétrica permite que uma técnica similar à utilizada na obtenção de (11), que envolve o limitante da união, seja utilizado para a obtenção de um limitante superior para a probabilidade de erro de símbolo. É possível mostrar que [3]

$P_{s}(e) \leq 4 \frac{\sqrt{M}-1}{\sqrt{M}} E_{u, \xi}\left[Q\left(\sqrt{2 \gamma_{2}}+\sqrt{\frac{2 \gamma_{2}}{\beta_{2}}} \frac{u}{\mu_{u}} \cos \xi\right)\right]$

onde

$$
\begin{aligned}
& \gamma_{2}=\gamma \frac{3}{2(M-1)} \\
& \beta_{2}=\beta \frac{3}{2(M-1)}
\end{aligned}
$$

com $\gamma$ e $\beta$ definidos por (9) e (10), respectivamente. Uma vantagem da utilização dos limitantes em (11) e (14) é que ambos estão expressos na mesma forma de (7). Assim, os limitantes superiores para a probalilidade de erro no caso MPSK e no caso MQAM podem ser todos obtidos a partir da determinação da probabilidade de erro de símbolo associada à deteção de sinais BPSK em presença de interferências, que será examinado na próxima seção.

\section{CÁlCULO DA PROBABILIDADE DE ERRO DE SÍMBOLO EM PRESENÇA DE INTERFERÊN- CIA CIRCULARMENTE SIMÉ- TRICA}

Observe que tanto a probabilidade de erro em (7) quanto os limitantes em (11) e (14) são proporcionais ao valor esperado da função

$$
f(w)=Q\left(\sqrt{2 \gamma}+\sqrt{\frac{2 \gamma}{\beta}} w\right)
$$

onde $w$ é a variável aleatória

$$
w=\frac{u}{\mu_{u}} \cos \xi
$$

Assim, as probabilidades de erro de símbolo (ou limitantes superiores) podem ser determinadas a partir do valor esperado de $f(w)$ com relaç ão a $w$, ou seja, a partir de

$$
E_{w}[f(w)]=\int_{-\infty}^{\infty} f(W) p_{w}(W) d W
$$

A integral acima requer o conhecimento da função densidade de probabilidade de $w$, usualmente não disponível. No entanto, o cálculo desta integral pode ser feito de maneira aproximada através, por exemplo, do método que utiliza regras de quadratura de Gauss e os momentos da variável aleatória $w$ [4]. Para a obtenç ão dos momentos da variável aleatória $w$ em (18) note que, como $u$ e $\xi$ são estatisticamente independentes,

$$
E\left[w^{k}\right]=E\left[\left(\frac{u}{\mu_{u}}\right)^{k}\right] E\left[\cos ^{k} \xi\right]
$$

Sendo $\xi$ uma variável aleatória uniformemente distribuída em $(0,2 \pi]$, tem-se que

$$
E\left[\cos ^{k} \xi\right]= \begin{cases}2^{-k}\left(\begin{array}{c}
k \\
\frac{1}{2} k
\end{array}\right) & ; k \text { par } \\
0 & ; k \text { i mpar }\end{cases}
$$

e conseqüentemente os momentos de $w$ dependem apenas da obtenção dos momentos da amplitude normalizada da interferência

$$
z=\frac{u}{\mu_{u}}
$$

A determinação analítica dos momentos de $z$ é uma tarefa complexa e praticamente impossível em situações gerais, ficando restrita a um número limitado de situações bastante particulares [5]. Uma alternativa para a soluçâo deste problema é a determinação dos momentos de $z$ através de simulação em computador $[7,6]$. Entretanto, a determinaç ão dos momentos de $z$ através de simulação pode, dependendo da situação, requerer um tempo de computação excessivo, além de exigir uma nova simulação para cada nova situaç ão específica. Por estes motivos, apesar do método descrito que utiliza a regra de quadratura de Gauss poder fornecer aproximaç ões muito boas para a probabilidade de erro de símbolo em presença de interferências, a sua utilização de forma ampla pode requerer um tempo de computação inadequado para algumas aplicaç ões como, por exemplo, o dimensionamento e otimização de transponders, que geralmente demanda repetidas análises do sistema, envolvendo diversas situações possíveis. Neste caso a utilização de limitantes superiores é mais adequada.

\subsection{Limitante de Glave e Rosembaum}

Dentre os limitantes superiores disponíveis na literatura, o proposto por Glave and Rosembaum [1, 2] é bastante conveniente, já que admite uma multiplicidade de tipos de interferências e, apesar de requerer apenas o conhecimento do valor de pico e do valor médio quadrático das amostras dos diversos sinais interferentes, é razoavelmente justo em diversas situações de interesse. Este limitante é baseado em um conceito bastante simples que consiste em se determinar, dentre as possíveis funções distribuição de probabilidade associadas à amplitude de uma interferência que tenha um dado valor rms 
e um dado valor de pico, aquela que produz a maior probabilidade de erro. A aplicação deste limitante para o cálculo da probabilidade de erro de símbolo em (7) (sinais BPSK), é descrita a seguir.

Considere a probabilidade de erro de símbolo para sinais BPSK em presença de interferência circularmente simétrica, dada por (7). Esta expressão pode ser reescrita como

$$
P_{s}(e)=E_{z, \xi}\left[Q\left(\sqrt{2 \gamma}+\sqrt{\frac{2 \gamma}{\beta}} z \cos \xi\right)\right]
$$

onde $z$ é a amplitude da interferência, normalizada, dada por (22).

O valor esperado em (23) pode ser efetuado tomando-se inicialmente o valor esperado com relação a $\xi$ e em seguida com relaç ão a $z$. Para tal é conveniente que se expresse a função $Q$ ( ) em (23) em série de Taylor em torno de $\sqrt{2 \gamma}$, tomandose em seguida o valor esperado com relação a $\xi$. Levando-se ainda em conta que $z$ e $\xi$ são estatisticamente independentes e (21), é possível mostrar que[1]

$$
P_{s}(e \mid z)=Q(\sqrt{2 \gamma})+\Sigma(z, \gamma, \beta)=q(z)
$$

onde

$$
\Sigma(z, \gamma, \beta)=\frac{e^{-\gamma}}{\sqrt{\pi}} \sum_{k=1}^{\infty} \frac{H_{2 k-1}(\sqrt{\gamma})}{2^{2 k} k ! k !}\left[\frac{\gamma}{\beta}\right]^{k}[z]^{2 k}
$$

com $H_{k}($ ) denotando o polinômio de Hermite de ordem $k$ [8]. Tem-se portanto que a probabilidade de erro não condicionada é dada por

$$
P_{s}(e)=E_{z}\left[P_{s}(e \mid z)\right]=\int_{0}^{\infty} q(Z) d F(Z)
$$

onde $F$ ( ) é a função distribuição de probabilidade de $z$.

O que se deseja é determinar a função $F($ ) que maximiza o funcional em (26), sujeito à restrição de potência da interferência $\left(E\left[u^{2}\right] \leq \mu_{u}^{2}\right)$, ou seja

$$
E\left[z^{2}\right]=\int_{0}^{\infty} Z^{2} d F(Z) \leq 1
$$

e à restrição de valor de pico da amplitude da interferência $\left(u \leq U_{p}\right)$, ou seja

$$
\int_{Z_{i^{+}}^{+}}^{\infty} d F(Z)=0
$$

onde $Z_{p}$ é o fator de pico (pf) da interferência, dado por

$$
Z_{p}=\frac{U_{p}}{\mu_{u}} \geq 1
$$

Se $F_{o}$ ( ) é a função que maximiza o funcional em (26), sujeita às restrições (27) e (28), então para qualquer interferência cuja amplitude tenha valor de pico $U_{p}$ e potência $\mu_{u}^{2}$ temse

$$
P_{s}(e) \leq \int_{0}^{\infty} q(Z) d F_{o}(Z)
$$

A integral do lado direito de (30) define o limitante proposto por Glave e Rosembaum em [1].

88
De acordo com os resultados em [1], a função $F_{o}($ ) existe e sua utilização em em (30), com $q$ ( ) dada por (24), fornece o limitante

$$
P_{s}(e) \leq L\left(Z_{\text {sup }}, \gamma, \beta\right)
$$

com

$$
\begin{aligned}
& L\left(Z_{\text {sup }}, \gamma, \beta\right)= \\
& \begin{cases}Q(\sqrt{2 \gamma})+\frac{1}{Z_{\text {sup }}^{2}} \Sigma\left(Z_{\text {sup }}, \gamma, \beta\right) & ; \quad Z_{\text {sup }} \geq 1 \\
Q(\sqrt{2 \gamma})+\Sigma(1, \gamma, \beta) & ; \quad Z_{\text {sup }}<1\end{cases}
\end{aligned}
$$

e onde $Z_{\text {sup }} \in\left[0, Z_{p}\right]$ é o valor de $Z$ que maximiza a funç ão

$$
g(Z)=\frac{1}{Z^{2}} \Sigma(Z, \gamma, \beta)
$$

ou seja,

$$
Z_{\text {sup }}=\max _{0 \leq Z \leq Z_{1},}^{-1} g(Z)
$$

Conforme ressaltado em [1], a função $g$ ( ) dada por (33) é uma função não decrescente em $Z$ até um determinado valor $Z_{\max }$ que, na maioria dos casos de interesse prático, excede o fator de pico $Z_{p}$ da interferência. Neste caso, resulta portanto de (34) que $Z_{\text {sup }}=Z_{p}$, e conseqüentemente

$$
P_{s}(e) \leq L\left(Z_{p}, \gamma, \beta\right) \quad ; \quad Z_{p} \geq 1
$$

Condições para que $Z_{\text {max }} \geq Z_{p}$ podem ser encontradas em [1], onde curvas deste limitante para portadoras BPSK, parametrizadas pelo valor do fator de pico do agregado de sinais interferentes são apresentadas.

Limitante para PSK M-ário

A extensão dos resultados apresentados para o caso $M>2$ é imediata. Uma simples comparação entre (7) e (11) levandose (31) em consideração permite escrever, para o caso MPSK $(M>2)$

$$
P_{s}(e) \leq 2 L\left(Z_{s u p}, \gamma_{1}, \beta_{1}\right)
$$

onde $L\left(\right.$, , ) é dado por (32), $\gamma_{1}$ e $\beta_{1}$ por (12) e (13) respectivamente e, de acordo com (33) e (34)

$$
Z_{\text {sup }}=\max _{0 \leq Z \leq Z_{\nu}}^{-1} \frac{1}{Z^{2}} \Sigma\left(Z, \gamma_{1}, \beta_{1}\right)
$$

$\operatorname{com} \Sigma(,,$,$) definida em (25). Curvas deste limitante para por-$ tadoras QPSK e PSK-8, parametrizadas pelo valor do fator de pico do agregado de sinais interferentes, podem ser encontradas em [3].

\section{Extensão ao caso QAM M-ário}

O uso do limitante de Glave e Rosembaum foi estendido ao caso de portadoras QAM. Isto foi feito aqui a partir da obtenção de um limitante superior conveniente para a probabilidade de erro de símbolo em presença de interferência circularmente simétrica. A determinaç ão deste limitante foi feita na forma descrita em [3] sendo o resultado dado por (14), (15) e (16). A conveniencia deste limitante superior é evidente quando se compara (14) com a probabilidade de erro de símbolo para sinais BPSK dada em (7). Esta comparação, 
levando-se (31) em consideração, permite obter diretamente para o caso MQAM o limitante superior expresso por

$$
P_{s}(e) \leq 4 \frac{\sqrt{M}-1}{\sqrt{M}} L\left(Z_{\text {sup }}, \gamma_{2}, \beta_{2}\right)
$$

onde $L(,,$,$) é dado por (32), e \gamma_{2}$ e $\beta_{2}$ dados por (15) e (16), com $\gamma$ e $\beta$ representando, respectivamente a razão portadoraruído (cnr) e a razão portadora-interferência (cir), definidos por (9) e (10). Ainda em (38) tem-se, de acordo com (33) e (34),

$$
Z_{\text {sup }}=\max _{0 \leq Z \leq Z_{p}}{ }^{-1} \frac{1}{Z^{2}} \Sigma\left(Z, \gamma_{2}, \beta_{2}\right)
$$

com $\Sigma($, , ) definida em (25). Um exemplo do limitante para o caso de portadoras QAM-16 é apresentado na Figura 2. Nesta figura, as quantidades CNR, CIR e PF correspondem às quantidades cnr, cir e pf, expressas em decibéis. Para efeito de comparação, esta figura apresenta também a curva relativa à aproximação Gaussiana, que corresponde à probabilidade de erro de símbolo que seria obtida considerando-se a interferência como sendo Gaussiana (curva tracejada).

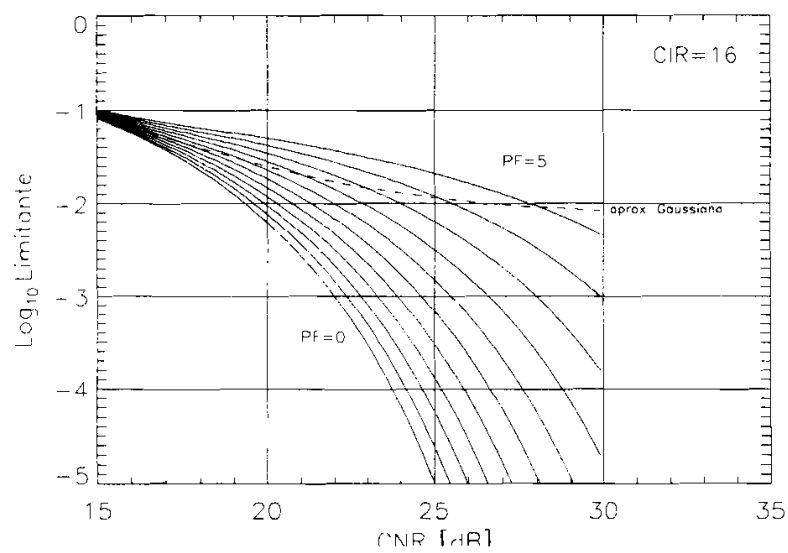

FIG. 2: Um exemplo da extensão do Limitante de Glave e Rosembaum para portadora vítima QAM-16.

Observe que a Figura 2 evidencia a existência de regiões onde a utilização da aproximação Gaussiana resultaria em um dimensionamento de enlace bem mais conservador do que aquele baseado no limitante apresentado. Considerando-se, por exemplo, uma probabilidade de erro de símbolo de $10^{-2}$, obtem-se da Figura 2, para $\mathrm{CIR}=16 \mathrm{~dB}$ e um fator de pico (PF) igual a $3 \mathrm{~dB}$, que a aproximação Gaussiana resulta em um valor requerido para CNR mais do que $5 \mathrm{~dB}$ acima do que seria requerido utilizando-se o limitante apresentado.

\subsection{Determinação do Fator de Pico de Si- nais Digitais Interferentes}

Conforme ressaltado na Seção 4.1., a obtenção do limitante de Glave e Rosembaum requer a determinaç ão do valor de pico das amostras dos diversos sinais interferentes. A Seção 8. do Apêndice formula matematicamente $o$ algoritmo proposto em $[1,2]$ para a determinação analí tica do valor de pico de sinais PSK M-ários filtrados. Este algoritmo, entretanto, não se aplica ao caso mais complexo de sinais interferentes QAM
M-ários. Um algoritmo original para a determinação do valor de pico de sinais QAM-16 filtrados foi então desenvolvido e é apresentado na Seção 8. do Apêndice.

Ressalte-se que o limitante de Glave e Rosembaum se aplica diretamente ao caso em que a interferência presente no sistema é formada por uma soma de interferências circularmente simétricas e estatisticamente independentes, uma vez que o resultado desta soma é também circularmente simétrica. Na Seção 8. do Apêndice são apresentadas expressões que permitem a obtenç ão do fator de pico de um agregado de interferências a partir do fator de pico de cada uma das parcelas deste agregado.

\section{PRECISÃo do LIMITANTE DE GLAVE E ROSEMBAUM}

Nesta seção, resultados obtidos através do limitante de Glave e Rosembaum para algumas situações de interesse prático são comparados a resultados obtidos utilizando-se o programa ASTRAL [6] que implementa o método que utiliza os momentos das amostras dos sinais interferentes e a regra de quadratura de Gauss na determinação da probabilidade de erro de sí mbolo, mencionado na Seção 4 ..

Para cada uma das situações consideradas, foram obtidas curvas de desempenho que indicam a probabilidade de erro de símbolo $P_{s}(e)$ versus $E_{b} / N_{0}$, para valores fixados da razão $I N R=\gamma / \beta$, entre as potências da interferência e do ruído após o filtro de recepção, com $\gamma$ e $\beta$ definidos por (9) e (10).

As curvas relativas ao limitante de Glave e Rosembaum foram inicialmente obtidas em função da quantidade $\gamma$, ulilizando-se as expressões apresentadas na Seção 4.1.. A conversão de $\gamma$ para $E_{b} / N_{0}$ foi feita utilizando-se a relação

$$
\gamma=\frac{E_{b}}{N_{0}} k R_{b}
$$

obtida a partir de (9). Nesta relação, a quantidade $k$ se escreve

$$
k=\frac{p_{0}^{2}}{\int_{-\infty}^{\infty}|H(f)|^{2} d f}
$$

onde $H(f)$ denota a resposta em frequiência do filtro de recepç ão $h(t)$ e $R_{b}$ é a taxa de transmissão em bit/s. É possí vel mostrar que se o filtro de recepção é casado ao pulso de transmissão e a amostra $p_{0}$ corresponde ao valor máximo do módulo do pulso $p(t)$, o produto $k R_{b}$ atinge seu valor máximo dado por $k R_{b}=\log _{2} M$, onde $M$ é o número de pontos da constelação de sinais da modulação utilizada. Neste caso $\gamma$ representa, portanto, a razão sinal-ruído por símbolo, $E_{s} / N_{0}$.

Em todas as situações consideradas, os pulsos de transmissão, tanto da portadora desejada quanto da portadora interferente, têm espectro em raiz quadrada de cosseno levantado com fator de roll-off $\rho$, e os filtros de recepção são casados aos pulsos de transmissão da portadora desejada. Na determinação das curvas do limitante de Glave e Rosembaum, os valores dos fatores de pico dos sinais interferentes filtrados foram obtidos utilizando-se os algoritmos mencionados na Seção 4.2. e apresentados no Apêndice.

Foram consideradas 3 situações, cujos resultados são apresentados nas figuras 3,4 , e 5 , respectivamente. Nestas figuras, 
$\bar{R}_{b}, \bar{R}_{s}$ e $\overline{\Delta f}$ denotam, respectivamente, os valores da taxa de transmissão, da taxa de símbolos e da diferença entre as frequiências das portadoras desejada e interferente, normalizados com relação à taxa de símbolos da portadora desejada.

Observa-se que, em todos os casos examinados, os resultados fornecidos pelos dois métodos considerados foram bastante próximos, com a diferença entre eles aumentando ligeiramente com o aumento do nível de interferência presente no sistema, o que seria de se esperar. É interessante notar que em alguns dos casos examinados os resultados obtidos com o programa ASTRAL situaram-se ligeiramente acima do limitante superior teórico obtido com a técnica de Glave e Rosembaum. Um exame dos procedimentos e expressões utilizadas em cada um dos métodos indicou que esta aparente anomalia deve-se, possivelmente, a problemas de precisão numérica resultantes da implementação, em computador digital, do método que utiliza a regra de quadratura da Gauss.

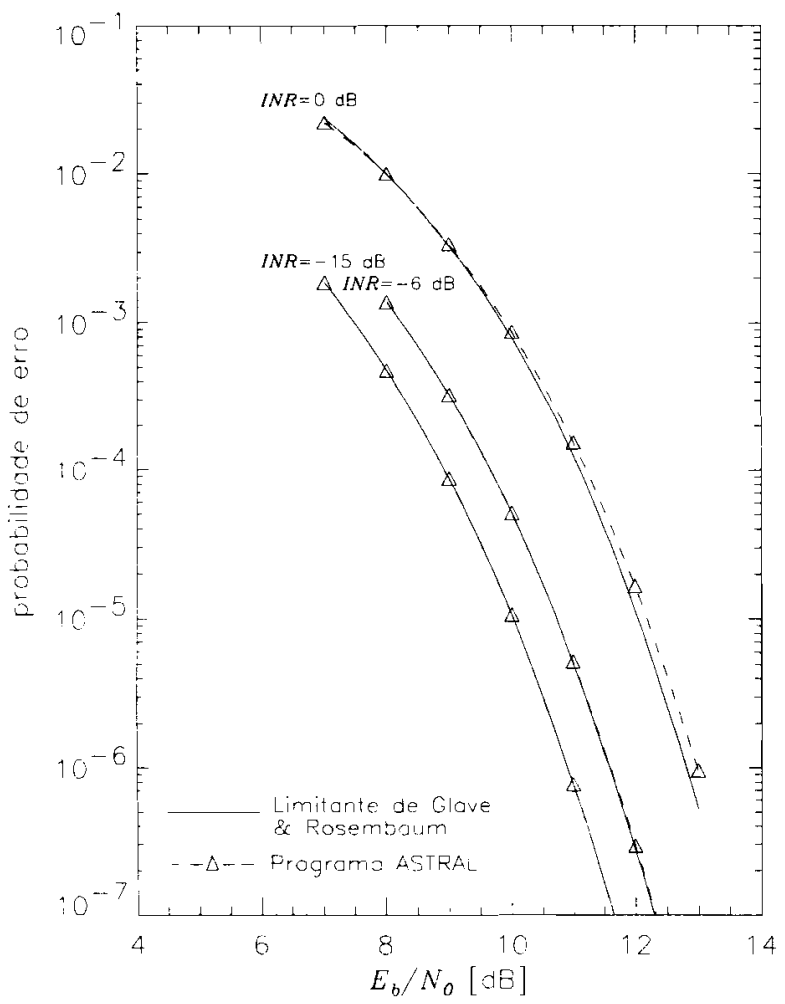

Fig. 3: Portadora desejada: QPSK, $\bar{R}_{b}=2, \bar{R}_{s}=1, \rho=$ 0,4 ; Portadora interferente : QPSK, $\bar{R}_{b}=4, \bar{R}_{s}=2, \rho=$ $0,4, \overline{\Delta f}=0,5$.

\section{CONSIDERAÇÃO DOS EFEITOS DE INTERFERÊNCIAS NA TAXA DE ERRO DE BIT DO SISTEMA}

Esta seção considera os efeitos de interferências externas na taxa de erro de bit (BER) de sistemas de transmissão digital levando em consideração a curva de desempenho do modem utilizado no sistema considerado, que pode ou não utilizar códigos corretores de erro (FEC). Em particular, o obje90

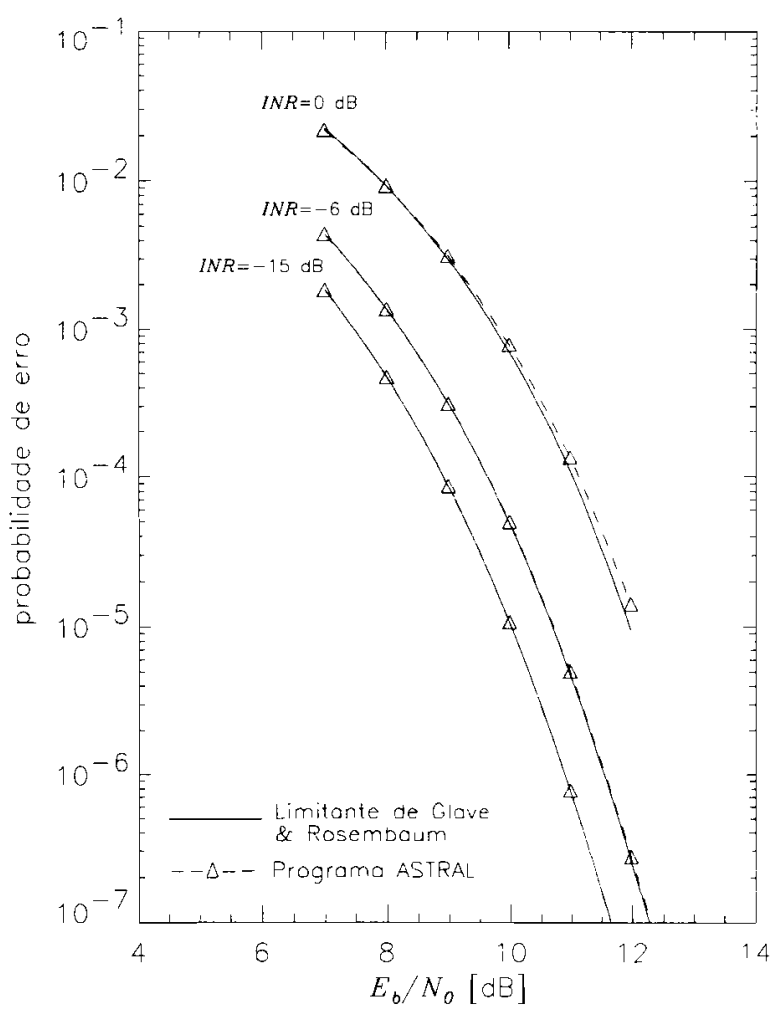

FIG. 4: Portadora desejada : QPSK, $\bar{R}_{b}=2, \bar{R}_{s}=1, \rho=$ 0,4 ; Portadora interferente : QAM-16, $\bar{R}_{b}=4, \bar{R}_{s}=1$, $\rho=0,4, \overline{\Delta f}=0$.

tivo aqui é a obtenç ão de uma estimativa da degradação na BER, na saída do modem, provocada pela presença de sinais interferentes. A análise deste problema resultou na proposição de uma estratégia para a avaliação desta degradação que é baseada num novo conceito de ruído térmico equivalente [3]. Em contraste com a estratégia usual que considera como ruído equivalente um ruído térmico que possua a mesma potência que a interferência presente na saída do filtro de recepção, a estratégia proposta define como ruído equivalente um ruído térmico que, colocado na entrada do receptor, conduza à mesma probabilidade de erro de símbolo (na entrada do decodificador) que a combinação de ruído e interferências presente no sistema. As figuras 6 e 7 ilustram, respectivamente, o conceito de ruído térmico equivalente proposto e o conceito usualmente adotado.

Observe na Figura 6 que o ruído equivalente $n_{e q}(t)$ colocado na entrada do receptor é aquele que produz, na entrada do decodificador, a mesma probabilidade de erro de símbolo $P_{s}(e)$ que aquela provocada pela combinação de ruído e interferência presente no sistema. A este ruído térmico equivalente corresponde, na saída do decodificador, uma probabilidade de erro de bit $\hat{P}_{b}(e)$ que é usada como uma estimativa da probabilidade de erro de bit $P_{b}(e)$ do sistema real. Por outro lado, conforme ilustrado na Figura 7, o ruído térmico equivalente, em sua definição usual, é aquele que gera na saída do filtro de recepção um ruído de potência igual à potência do combinado de ruído térmico e interferência na saída deste mesmo filtro. Neste caso, a probabilidade de erro de símbolo $P_{s}^{\prime}(e)$ pro- 


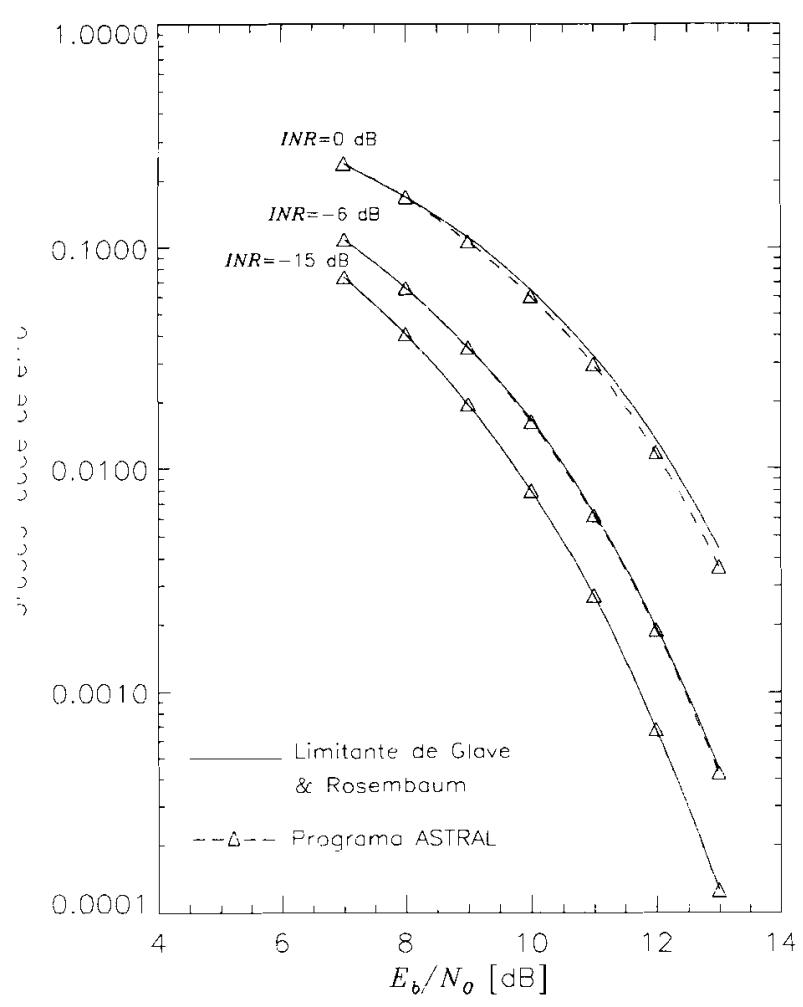

FIG. 5: Portadora desejada : QAM-16, $\bar{R}_{b}=4, \bar{R}_{s}=1$, $\rho=0,4$; Portadora interferente : QPSK, $\bar{R}_{b}=2, \bar{R}_{s}=1$, $\rho=0,4, \overline{\Delta f}=0$.

vocada por este ruído equivalente na entrada do decodificador difere da do sistema real sendo, em geral, superior. Desse modo, a estimativa da probabilidade de erro de bit $P_{b}^{\prime}(e)$ na saída do decodificador obtida através desta última estratégia é, em geral, mais pessimista e menos confiável do que aquela obtida com a estratégia proposta.

Conforme ilustrado através da Figura 8, o procedimento para a obtenção de estimativas da probabilidade de erro de bit (BER) através da estratégia proposta pode ser feita utilizandose três tipos de curvas de desempenho :

(i) a curva de probabilidade de erro de símbolo associada à modulação e aos tipos de filtro de transmissão e deteç ão utilizados, considerando-se o ruído térmico e as interferências presentes no sistema. Esta curva pode ser estimada a partir das expressões para o limitante de Glave e Rosembaum, apresentadas na Seção 4.1., e das equações (40) e (41);

(ii) a curva de probabilidade de erro de símbolo associada à modulação e aos tipos de filtro de transmissão e deteç ão utilizados, considerando-se apenas a presença de ruído térmico. Esta curva pode ser obtida a partir das expressões da Seç ão 3., $\operatorname{com} \beta=\infty$, e das equações (40) e (41); e

(iii) a curva de desempenho do modem, em termos de BER, em presença de ruído térmico (fornecida pelo fabricante).

Nesta figura, considera-se a situação em que um sistema

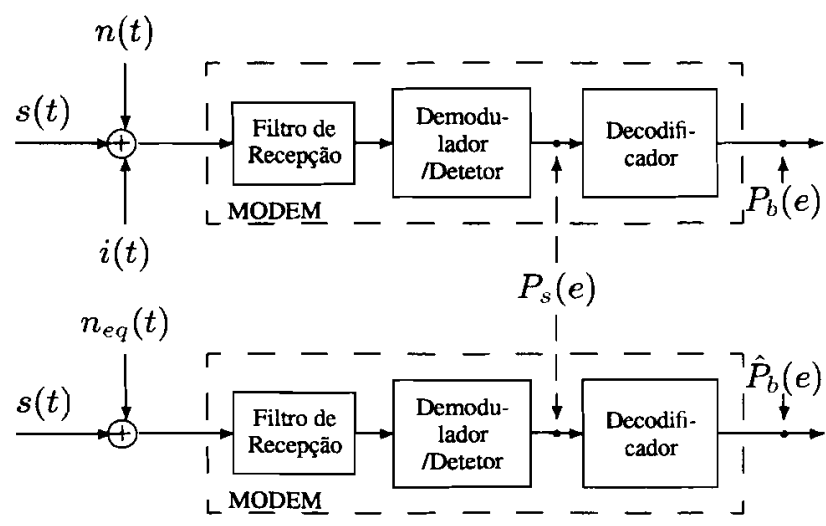

FIG. 6: Conceito de ruído térmico equivalente proposto.
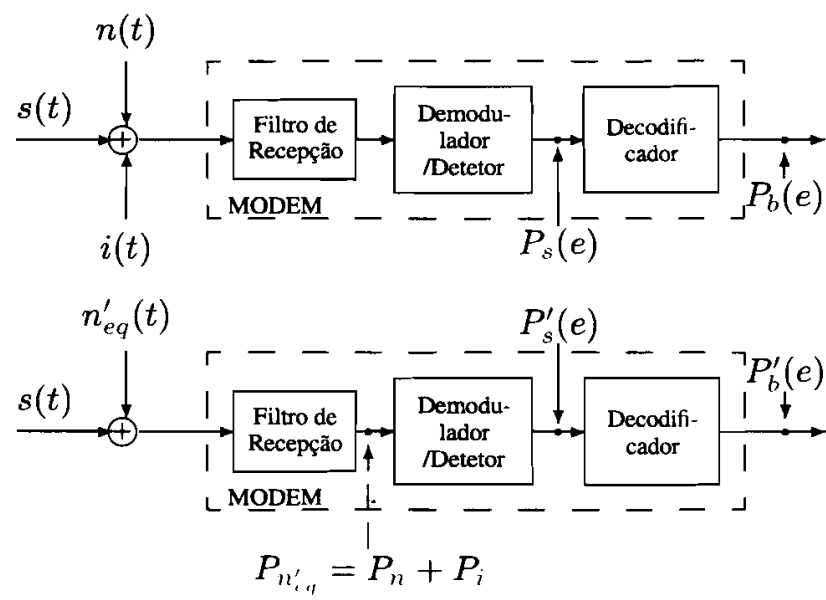

FIG. 7: Conceito de ruído térmico equivalente usualmente empregado.

de transmissão digital que utiliza FEC está operando em presença de interferência circularmente simétrica caracterizada (na saída do filtro de deteção) por um fator de pico de $1 \mathrm{~dB}$ e um nível de potência $3 \mathrm{~dB}$ abaixo da potência do ruído (INR $=\gamma / \beta=-3 \mathrm{~dB})$. A título de exemplo, suponha que se deseja estimar a BER deste sistema para um dado valor de $E_{b} / N_{0}(6,7 \mathrm{~dB})$. Para este valor, obtem-se pelo limitante de Glave e Rosembaum probabilidade de erro de símbolo do sistema (aproximadamente $3 \times 10^{-3}$ ). De acordo com a curva de desempenho da modulação, este valor de probabilidade de erro seria provocado por um ruído térmico equivalente correspondente a um dado valor de $E_{b} / N_{0_{c^{\prime \prime}}}(5 \mathrm{~dB})$. Note que o valor de $E_{b}$ nesta razão corresponde à energia por bit transmitido. Utilizando-se a taxa do FEC do modem chega-se ao valor da razão $E_{B} / N_{0 \ldots,}$, onde $E_{B}$ representa a energia por bit de informaç ão. Finalmente o valor desta razão é levado à curva de desempenho do MODEM, fornecendo a estimativa desejada do valor da $B E R\left(10^{-6}\right)$.

Note que este procedimento permite que se obtenha curvas de desempenho do modem (BER versus $E_{B} / N_{0}$ ) parametrizadas por valores da razão interferência-ruído $(I N R)$, ou da razão portadora-interferência $(C / I)$.

Muitas vezes, as situações de interesse dizem respeito à determinação do $E_{b} / N_{0}$ requerido para uma dada taxa de erro de bit. Neste caso, considerando-se a Figura 8 e supondo-se 


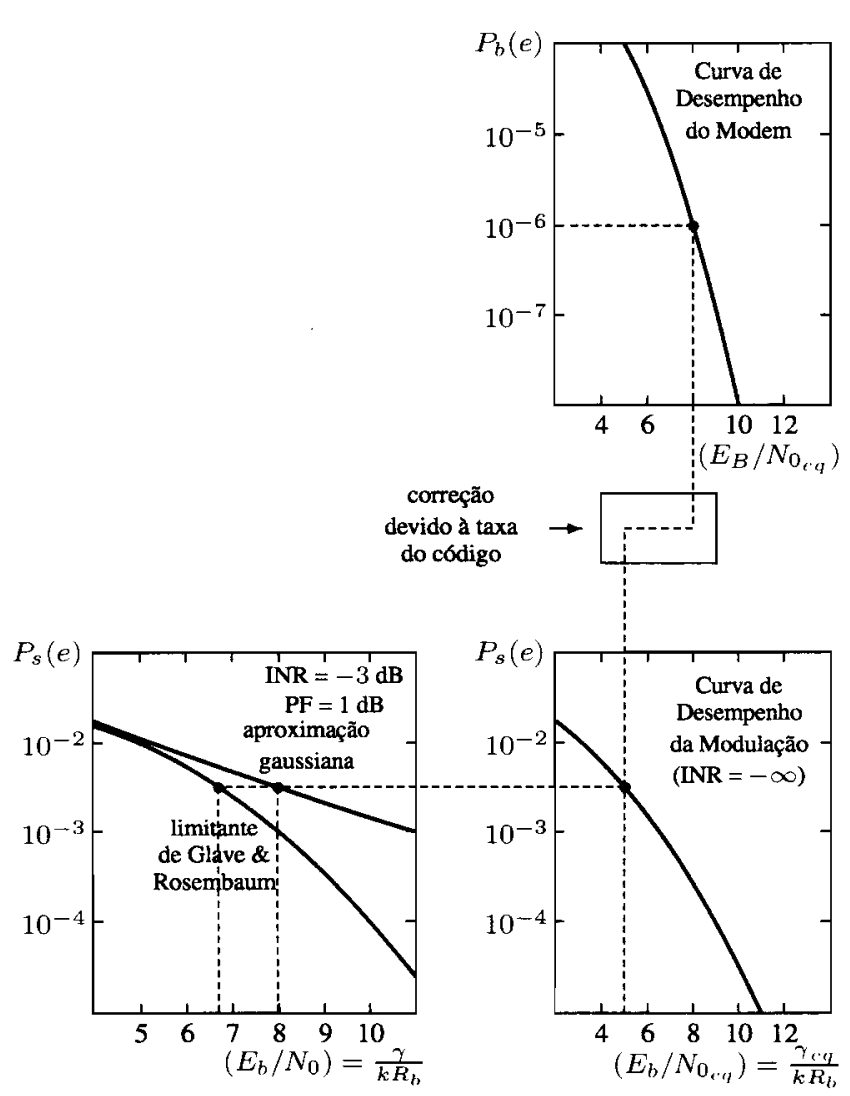

FIG. 8: Consideração dos efeitos de interferências na BER de sistemas de transmissão digital, utilizando-se o conceito de ruído térmico equivalente proposto.

como objetivo uma $B E R$ de $10^{-6}$ o procedimento inverso, a partir da curva de desempenho do MODEM, conduziria a um $E_{b} / N_{0}$ requerido de $6,7 \mathrm{~dB}$. Observe-se que a utilização da aproximação gaussiana para a caracterizaç ão da interferência presente no sistema exigiria, neste exemplo, um valor de $E_{b} / N_{0}$ igual a $8 \mathrm{~dB}$.

\section{EXEMPLO DE APLICAÇÃO A UMA SITUAÇÃO PRÁTICA DE INTERESSE}

Os procedimentos descritos nas seções anteriores foram aplicados para avaliar os efeitos de interferências mútuas entre sistemas do serviço Ponto-Multiponto (PMP) da Embratel e o enlaces do sistema rádio digital RADI-234. A Figura 9 ilustra a situação particular examinada envolvendo a possibilidade de utilização de uma portadora PMP centrada em $2116,5 \mathrm{MHz}$ e portadora RADI-234 centrada em $2135 \mathrm{MHz}$. Neste caso, a densidade espectral de potência (DEP) da portadora QPSK RADI-234 foi obtida considerando-se um pulso de transmissão gerado através da passagem de um pulso retangular por um filtro de Butterworth de 3 polos, com produto $B T=0,5$, a uma taxa de 17,184 Msímbolo/s. O filtro de recepção da portadora RADI-234 foi considerado de Butterworth com 5 polos e $B T=0,95$. Estes dados, juntamente com a curva de desempenho do modem em presença 92 de ruído térmico, foram ajustados a partir de informações recebidas do CPqD-Telebrás. Para a DEP da portadora QPSK do serviço PMP, considerou-se que o pulso de transmissão é gerado através da passagem de um pulso retangular por um filtro de Butterworth com 5 polos, com produto $B T=0,9$, a uma taxa de 2,496 Msímbolo/s. O filtro de recepção da portadora PMP foi considerado de Butterworth com 5 polos e $B T=0,9$. Estes dados, juntamente com a curva de desempenho do modem em presença de ruí do térmico, foram ajustados a partir de características técnicas fornecidas pela NEC. Neste exemplo particular, nenhum dos dois sistemas utiliza FEC.

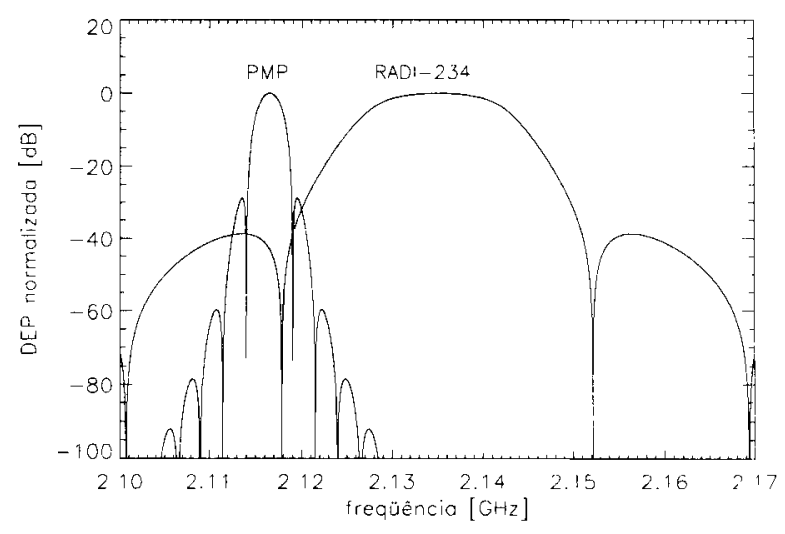

FIG. 9: Densidade Espectral de Potência Normalizada.

Foi examinado tanto o efeito da portadora RADI-234 sobre a portadora do serviço PMP quanto o efeito da portadora PMP sobre a portadora RADI-234. No primeiro caso (interferência sobre a portadora do serviço PMP) foi considerado o desempenho do enlace Terminal-Nodal. Os resultados obtidos estão contidos na Figura 10 que apresenta curvas de desempenho da portadora PMP em presença de portadora interferente RADI234 expressas em função da razão portadora-ruído $(C / N) \mathrm{e}$ do nível de sinal recebido $(R S L)$, parametrizadas pela razão portadora-interferência $(C / I)$ na entrada do filtro de recepção.

O segundo caso examinado diz respeito aos efeitos da interferência produzida pela portadora PMP sobre a portadora RADI-234. Os resultados obtidos estão contidos na Figura 11 que apresenta o desempenho da portadora RADI-234 na presença da portadora PMP interferente expresso em função da razão portadora-ruído $(C / N)$ e do nível de sinal recebido $(R S L)$. Os resultados numéricos apresentados indicam que a utilização, por portadoras do serviç o PMP, da frequiência $f=2.116,5 \mathrm{MHz}$ não provocaria degradaç ão significatica no desempenho da portadora PMP (para $B E R=10^{-7}$, por exemplo, resulta uma degradação de razão sinal-ruído menor que $0,8 \mathrm{~dB}$ para $C / I \geq 0 \mathrm{~dB}$ ). $\mathrm{O}$ caso de interferência sobre portadoras RADI-234 é entretanto mais crítico. Para uma $B E R=10^{-7}$, por exemplo, uma degradação menor do que $1 \mathrm{~dB}$ só é observada quando $C / I \geq 15 \mathrm{~dB}$. Para $C / I=0 \mathrm{~dB}$, resulta uma degradação de aproximadamente $6 \mathrm{~dB}$. Assim, os resultados apresentados indicam que a utilização de portadora PMP na freqüência mencionada torna-se 


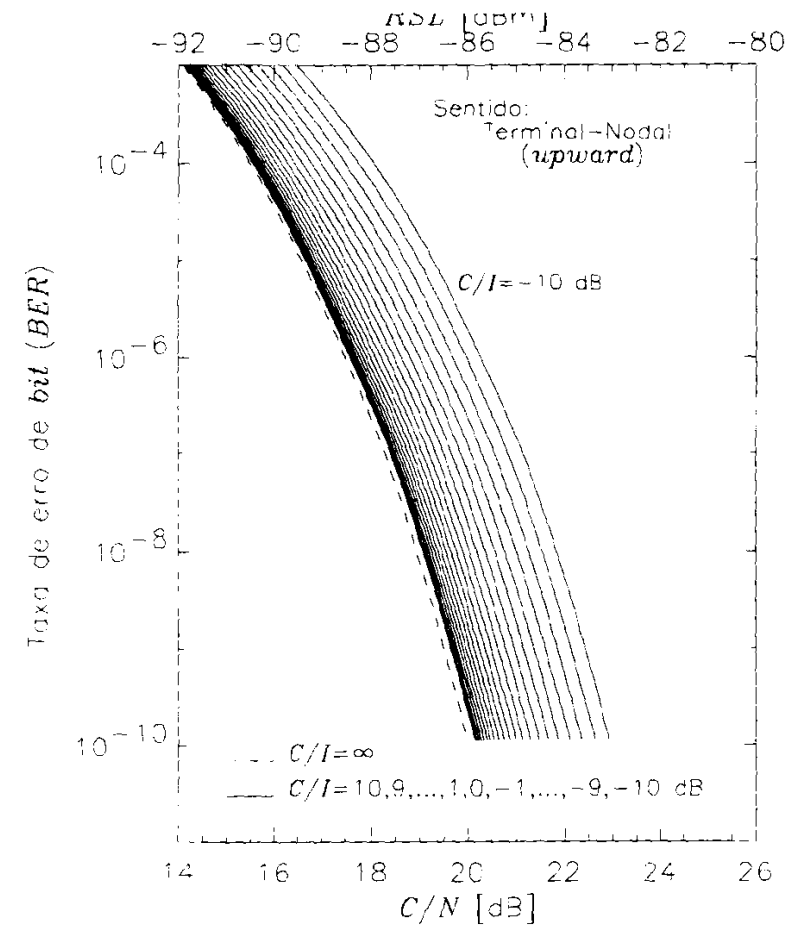

FIG. 10: Degradação na $B E R$ de portadora do Sistema PMP em 2116,5 MHz produzida por portadora do RADI-234 em $2135 \mathrm{MHz}$ (fator de reduç ão de interferência devido ao filtro de recepção : $23,72 \mathrm{~dB}$ ). No cálculo do nível de sinal recebido $(R S L)$ foi considerada uma Figura de Ruído de $3 \mathrm{~dB}$.

viável desde que os valores da razão portadora-interferência na entrada do receptor, associados ao sistema RADI-234, sejam suficientemente elevados $(C / I>15 \mathrm{~dB})$.

\section{CONCLUSÃO}

Os resultados obtidos indicam que a utilização do limitante de Glave e Rosembaum associada aos algoritmos para a determinaç ão analítica do valor de pico de portadoras digitais apresentados neste trabalho constitui uma ferramenta rápida e eficiente na determinação da probabilidade de erro de símbolo de sistemas de transmissão digital em presença de interferências, sem comprometer a precisão, mesmo em situações envolvendo altos níveis de interferência.

O conceito de ruído térmico equivelante utilizado neste trabalho permite a obtenção de estimativas da taxa de erro de bit do sistema a partir do conhecimento da probabilidade de erro de símbolo em presença de interferências e da curva de desempenho do modem em presença de ruído. Sendo assim, a estimativa obtida leva em consideração a possível utilização de códigos corretores de erro e as perdas de desempenho associadas à implementaç ão prática do modem como, por exemplo, perdas devidas ao sincroní smo de relógio, à recuperação de portadora, etc. As estimativas de $B E R$ obtidas através do conceito de ruído térmico equivalente aqui proposto, devido à sua própria definição, são mais confiáveis e, em geral, menos pessimistas do que aquelas obtidas considerando-se a interferência como um ruído térmico de mesma potência na saída do

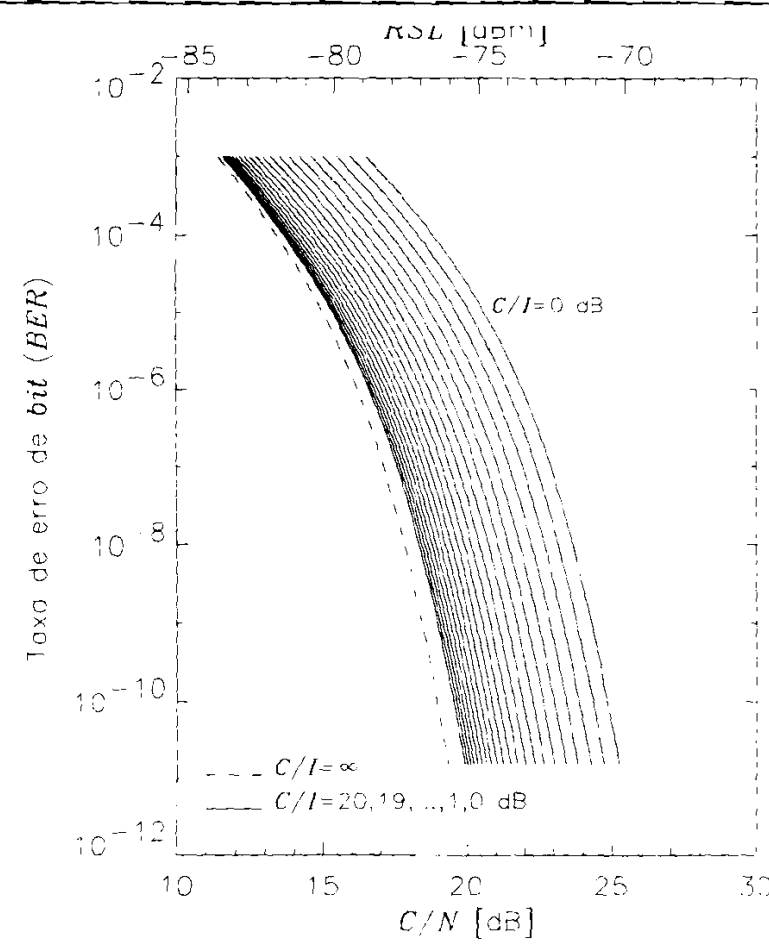

FIG. 11: Degradação na $B E R$ de portadora do RADI-2 em $2135 \mathrm{MHz}$ produzida por portadora do Sistema PMP $2116,5 \mathrm{MHz}$ (fator de reduç ão de interferência devido ao tro de recepção : 6,02 dB). No cálculo do nível de sinal re bido $(R S L)$ foi considerada uma Figura de Ruído de $5 \mathrm{~dB}$

filtro de recepção.

\section{APÊNDICE}

\section{DETERMINAÇÃO DO FATOR DE PICO DE SINA DIGITAIS INTERFERENTES}

Conforme ressaltado na Seção 4.1., a obtenção do limit de Glave e Rosembaum requer a determinação do fato pico das amostras dos diversos sinais interferentes. A $S_{i}$ 8. descreve o algoritmo proposto em $[1,2]$ para a dete nação analítica do valor de pico de sinais MPSK filtrঞ Um algoritmo para a determinação do valor de pico de $s$ QAM-16 filtrados foi desenvolvido e é apresentado na $S$ 8.. Finalmente na Seção 8. são apresentadas expressõe: permitem a obtenção do fator de pico da amostra de um gado de interferências a partir do fator de pico de cada das parcelas deste agregado.

\section{A. Valor de Pico de Portadoras MPSK}

Seja $\tilde{u}(t)$ a envoltória complexa do sinal interferente. caso particular uma portadora MPSK, filtrado pelo fi] recepç ão da portadora desejada. Esta envoltória comr amostrada de modo a gerar $N=2 L+1$ números com (vetores bi-dimensionais), dados por

$$
\tilde{u}_{i+L+1}^{\tau}=\tilde{u}\left(\tau+i T_{I}\right) ; \quad i=-L, \ldots, L
$$

Em (42), o valor de $L$ está associado ao número $N$ de : los consecutivos a serem considerados no cálculo do 
pico $\left(L=\frac{N-1}{2}\right)$, e $T_{I}$ é o inverso da taxa de símbolos $R_{I}$ do sinal interferente em consideração e $\tau$ é um instante qualquer pertencente ao intervalo $\left[0, T_{I}\right.$ ).

A partir do conjunto dos $N$ números complexos $\left\{\tilde{u}_{j}^{\tau}, j=\right.$ $1, \ldots, N\}$, obtem-se um outro conjunto contendo $M N$ números complexos, dados por

$$
\tilde{y}_{k N+j}^{\tau}=e^{j \frac{2 \pi k}{M !}} \tilde{u}_{j}^{\tau} ; \quad \begin{aligned}
& j=1, \ldots, N \\
& k=0, \ldots, M-1
\end{aligned}
$$

onde $M$ é o número de fases da modulação PSK considerada.

Os $M N$ elementos complexos $\left\{\tilde{y}_{n}^{\tau}, n=1, \ldots, M N\right\}$ do conjunto obtido são então ordenados em ordem crescente de fase, dando origem a um novo conjunto, ordenado, $\left\{\tilde{z}_{n}^{\tau}, n=\right.$ $1, \ldots, M N\}$ de números complexos. Com base neste novo conjunto de números complexos, é possivel definir a função $g(\ell, \tau)$, dada por

$$
g(\ell, \tau)=\left|\sum_{m=1+\ell}^{N+\ell} \tilde{z}_{m}^{\tau}\right| ; \quad \ell=0, \ldots, N-1
$$

O valor de pico $U_{p}$, associado à portadora MPSK considerada, pode finalmente ser encontrado através da relação

$$
U_{p}=\max _{\tau} f(\tau) ; \quad 0 \leq \tau<T_{I}
$$

onde

$$
f(\tau)=\max _{\ell} g(\ell, \tau) ; \quad \ell=0, \ldots, N-1
$$

\section{B. Valor de Pico de Portadoras QAM-16}

Seja $\tilde{u}(t)$ a envoltória complexa do sinal interferente, neste caso particular uma portadora QAM-16, filtrado pelo filtro de recepç ão da portadora desejada. Assim como na subseção anterior, esta envoltória complexa é amostrada de modo a gerar $N=2 L+1$ números complexos (vetores bi-dimensionais), dados por

$$
\tilde{u}_{i+L+1}^{\tau}=\tilde{u}\left(\tau+i T_{I}\right) ; \quad i=-L, \ldots, L
$$

Em (47), o valor de $L$ está associado ao número $N$ de símbolos consecutivos a serem considerados no cálculo do valor de pico $\left(L=\frac{N-1}{2}\right)$, e $T_{I}$ é o inverso da taxa de símbolos $R_{I}$ do sinal interferente em consideração e $\tau$ é um instante qualquer pertencente ao intervalo $\left[0, T_{I}\right)$.

A partir do conjunto dos $N$ números complexos $\left\{\tilde{u}_{j}^{\tau}, j=\right.$ $1, \ldots, N\}$, obtem-se um outro conjunto contendo $4 N$ números complexos, dados por

$$
\tilde{y}_{k N+j}^{\tau}=e^{j \frac{\pi k}{2}} \tilde{u}_{j}^{\tau} ; \quad \begin{aligned}
& j=1, \ldots, N \\
& k=0, \ldots, 3
\end{aligned}
$$

Os $4 N$ elementos complexos $\left\{\tilde{y}_{n}^{\tau}, n=1, \ldots, 4 N\right\}$ do conjunto obtido são então ordenados em ordem crescente de fase, dando origem a um novo conjunto, ordenado, $\left\{\tilde{z}_{n}^{\tau}, n=\right.$ $1, \ldots, 4 N\}$ de números complexos. Com base neste novo conjunto de números complexos e nos inteiros $L_{1}$ e $L_{2}$ definidos por

$$
\begin{cases}L_{1}= & \begin{array}{l}
\text { número de complexos } \tilde{z}_{n}^{\tau} \text { com fase } \\
\text { no intervalo }\left(0, \tan ^{-1}(1 / 3)\right]
\end{array} \\
L_{2}= & \begin{array}{l}
\text { número de complexos } \tilde{z}_{n}^{\tau} \text { com fase } \\
\text { no intervalo }\left(0, \tan ^{-1}(3)\right]
\end{array}\end{cases}
$$

é possível definir a função $g(\ell, \tau)$, dada por

$$
g(\ell, \tau)= \begin{cases}\left|\sum_{m=1+\ell}^{N+\ell} \tilde{z}_{m}^{\tau}\right| & ; \quad 0<\ell \leq L_{2} \\ \max _{\mathbf{a}_{\ell}\left|q\left(\ell, \tau, \mathbf{a}_{\ell}\right)\right|} \mid L_{1}<\ell \leq L_{2} \\ \left|\sum_{m=1+\ell}^{N+\ell} \tilde{z}_{m}^{\tau}\right| & ; \quad L_{2}<\ell \leq N\end{cases}
$$

onde a quantidade $q\left(\ell, \tau, \mathbf{a}_{\ell}\right)$ é dada por

$$
\begin{aligned}
& q\left(\ell, \tau, \mathbf{a}_{\ell}\right)=\sum_{m=k_{1,}+\ell+1}^{k_{2 \ell}+N} \tilde{z}_{m}^{\tau} \\
& +\sum_{m=1+\ell}^{k_{1,+\ell}+\ell}\left(\alpha_{m} \tilde{z}_{m}^{\tau}+\frac{\left(1-\alpha_{m}\right) \sqrt{5}}{3} \tilde{z}_{m}^{\tau} e^{j \Phi}\right) \\
& \left.+\sum_{\left.m=k_{2,2+\ell+1}+\alpha_{m}\right) \sqrt{5}}^{N+\ell} \tilde{z}_{m}^{\tau} e^{-j \Phi}\right)
\end{aligned}
$$

com

$$
\Phi=\frac{\pi}{4}+\tan ^{-1}\left(\frac{1}{3}\right)
$$

e

$$
\alpha_{j+\ell} \in\{0,1\} \quad ; \quad j=1, \ldots, k_{1 \ell}, k_{2 \ell}+1, \ldots, N
$$

Os inteiros $k_{1 \ell}$ e $k_{2 \ell}$ que aparecem em (51) e (53) são definidos por

$$
\begin{cases}k_{1 \ell}= & \begin{array}{l}
\text { número de complexos } \tilde{z}_{n}^{\tau} \text { com fase } \\
\text { no intervalo }\left(\angle \tilde{z}_{\ell}^{\tau}, \angle \tilde{z}_{\ell}^{\tau}+\beta\right]
\end{array} \\
k_{2 \ell}= & \begin{array}{l}
\text { número de complexos } \tilde{z}_{n}^{\tau} \text { com fase } \\
\text { no intervalo }\left(\angle \tilde{z}_{\ell}^{\tau}, \angle \tilde{z}_{\ell}^{\tau}+\pi / 2-\beta\right]
\end{array}\end{cases}
$$

com

$$
\beta=\frac{\pi}{4}-\tan ^{-1}\left(\frac{1}{3}\right)
$$

O vetor $\mathbf{a}_{\ell}$ em (50) e (51), de dimensão $k_{1 \ell}+N-k_{2 \ell}$, é definido por

$$
\mathbf{a}_{\ell}^{T}=\left(\alpha_{1+\ell}, \ldots, \alpha_{k_{1 \ell}+\ell}, \alpha_{k_{2 \ell}+\ell+1}, \ldots, \alpha_{N+\ell}\right)
$$

O valor de pico $U_{p}$, associado à portadora QAM-16 considerada, pode finalmente ser encontrado através da relação

$$
U_{p}=\max _{\tau} f(\tau) ; \quad 0 \leq \tau<T_{I}
$$

onde

$$
f(\tau)=\max _{\ell} g(\ell, \tau) ; \quad \ell=0, \ldots, N-1
$$

com $g(\ell, \tau)$ dado por (50). 


\section{Fator de Pico de um Agregado de Interferências}

O limitante de Glave e Rosembaum, conforme descrito na Seção 4.1., se aplica diretamente ao caso em que a interferência presente no sistema é formada por uma soma de interferências circularmente simétricas e estatisticamente independentes, uma vez que o resultado desta soma é também circularmente simétrica. Assim, para a obtenção do limitante é necessário determinar o fator de pico do agregado de interferências. Para isso note-se inicialmente que o valor de pico $U_{\text {pag }}$ de uma soma de interferências circularmente simétricas corresponde à soma dos valores de pico $U_{p}$, de cada uma das parcelas interferentes, ou seja,

$$
U_{p_{(u)}}=\sum_{i=1}^{n} U_{p_{i}}
$$

Por outro lado, devido à independencia estatística, o valor médio quadrático $\mu_{a g}^{2}$ do agregado de interferências é igual à somas dos valores médios quadráticos $\mu_{u}^{2}$ de cada uma das parcelas. Tem-se assim,

$$
\mu_{a g}^{2}=\sum_{i=1}^{n} \mu_{u_{i}}^{2}
$$

Resulta então que o fator de pico $Z_{p_{u g}}$ do agregado de interferências, definido por

$$
Z_{p_{a g}}=\frac{U_{p_{a l u}}}{\mu_{a g}}
$$

pode ser escrito em termos dos fatores de pico $Z_{p}$, de cada uma das parcelas na forma

$$
Z_{p_{n, u}}=\left(Z_{p_{k}}+\sum_{\substack{i=1 \\ i \neq k}}^{n} \lambda_{i} Z_{p,}\right)\left(\sqrt{1+\sum_{\substack{i=1 \\ i \neq k}}^{n} \lambda_{i}^{2}}\right)^{-1}
$$

onde

$$
\lambda_{i}=\sqrt{\frac{\mu_{u,}^{2}}{\mu_{u_{k}}^{2}}}
$$

Observe-se que, em (62) e (63), o índice $k$ representa o índice de uma parcela qualquer arbitrariamente tomada como referência.

\section{REFERÊNCIAS}

[1] A. S. Rosenbaum and F. E. Glave, An Error-Probability Upper Bound for Coherent Phase-Shift Keying with PeakLimited Interference, IEEE Transactions on Communications, vol. COM-22, no. 1, January 1974.

[2] F. E. Glave and A. S. Rosenbaum, An Upper Bound Analysis for Coherent Phase-Shift Keying with Cochannel, Adjacent-Channel, and Intersymbol Interference, IEEE Transactions on Communications, vol. COM-23, no. 6, June 1975.

[3] J. M. P. Fortes e R. Sampaio Neto, Avaliação dos Efeitos de Interfeências em Sistemas de Transmissão Digital, Relatório CETUC D-ST-01/96, Junho 1996.
[4] S. Benedetto, E. Biglieri and V. Castellani, Digital Transmission Theory, Prentice-Hall, Inc., Englewood Cliffs, New Jersey 07632, 1987.

[5] S. Benedetto, E. Biglieri and V. Castellani, Combined Effects of Intersimbol, Interchannel, and Co-channel Interferences in M-ary CPSK systems IEEE Transactions on Communications, vol. COM-21, September 1973.

[6] J. C. Brandão, ASTRAL : Um Programa para Análise e Simulação de Sistemas de Transmissão Digital, Relatório CETUC D-ST-05/87, janeiro 1987.

[7] E. L. Pinto and J. C. Brandão, On the Efficient Use of Computer Simulated Digital Signals to Evaluate Performance Paremeters, IEEE Journal on Selected Areas in Communications, vol. 6, no. 1, January 1988.

[8] I. S. Gradshteyn and I. M. Ryzhik, Table of Integrals, Series, and Products, Academic Press, New York, 1965.

José Mauro P. Fortes nasceu no Rio de Janeiro, em 25 de maio de 1950. Graduou-se em Engenharia Elétrica (Telecomunicações) na Pontifícia Universidade Católica do Rio de Janeiro (PUC-Rio) em 1973 e obteve o grau de Mestre em $\mathrm{Ci}$ ?ncias em Engenharia Elétrica na mesma universidade em 1976. Concluiu o doutorado na Stanford University, USA, em 1980, obtendo o título de Ph.D. em Engenharia Elétrica. Em junho de 1980 retornou à PUC-Rio onde é atualmente Professor Associado, trabalhando em ensino e pesquisa no Centro de Estudos em Telecomunicações da Universidade Católica (CETUC). Durante o ano de 1992, enquanto em licença sabática da PUC-Rio, foi pequisador do Ultrasound Group do General Electric Research and Development Center em Schenectady, NY, USA. Desde 1987 é Vice Presidente do Grupo de Estudos 4 do Setor de Radiocomunicações da União Internacional de Telecomunicações (ITU-BR), que trata do Serviço Fixo por Satélite. Desde 1996 é Presidente da Sociedade Brasileira de Telecomunicações. Seus principais interesses estão nas áreas de comunicaçōes por satélite, teoria de estimação e processamento digital de sinais.

Raimundo Sampaio Neto nasceu em Rezende, RJ, em 16 de junho de 1952. Formou-se em Engenharia Elétrica (Telecomunicações) na Pontifícia Universidade Católica do Rio de Janeiro (PUC-Rio) em 1975 e obteve o grau de Mestre em Ciências em Engenharia Elétrica na mesma universidade em 1978. Concluiu o doutorado na University of Southern California (USC) em Los Angeles em 1983, com o título de Ph.D. em Engenharia Elétrica. De 1983 a 1984 prestou serviços de consultoria em análise de sistemas de comunicações junto à Axiomatic Inc., em Los Angeles, e desenvolveu atividades de pesquisa na área de sincronização de códigos em sistemas spread spectrum, como Post-doctoral Fellow do Communication Sciences Institute da USC. Em julho de 1984 retornou à PUC-Rio onde é atualmente Professor Associado, trabalhando em ensino e pesquisa no Centro de Estudos em Telecomunicações da Universidade Católica (CETUC). Durante o ano de 1991, enquanto em licença sabática da PUCRio, foi Professor Visitante do Departamento de Engenharia Elétrica da USC. Suas principais áreas de interesse são comunicações via satélite, sincronização em sistemas digitais e processamento digital de sinais. 